\title{
Illusory Snakes Might Be Due to Asynchronized Respective Field Remapping
}

\author{
Ahmad Yousef ${ }^{1}$ \\ 'School of Computational Science and Engineering, McMaster University, Hamilton, Ontario, Canada \\ *Correspondence: mohamas2@mcmaster.ca
}

Abstract- In this proposal, we try to virtually navigate inside the human brain to understand the neural mechanism of the perception of illusory snakes. To achieve this mission, we have to imagine the neural network of the visual motion perception during spontaneous saccadic eye movements; and digging into clear distinction between the foveal versus the peripheral visual receptive field remapping. We had previously discussed that conscious perception generated by the central retina has very different attributes than the visual awareness generated by the peripheral retina. It was clear that the central retina triggers visual perception which decelerates the apparent motion of the cyclic elements, and enlarge the size of these elements, see reference 2 . The peripheral retina, however, not only accelerates the apparent motion, but it generates illusory motion reversals, see reference 19. Since there are clear discrepancies in the spatiotemporal characteristics between the central and the peripheral retina in the visual awareness, we hypothesized that the illusory rotating snakes might be due to asynchronized respective field remapping; namely, a rivalrous remapping processes of the central versus the peripheral retinal images. In another word, the respective field remapping process triggered by the central retina has different spatial and temporal feeds to the visual awareness than the retinal peripheries. Interestingly, it had been found that deactivating the retinal peripheries through significant reduction against the contrast of the stimulus (that may stop the retinal peripheries from signaling the brain) eliminates the rotating snakes illusion. Elimination that might evidence the role of active retinal peripheries in creating the perception of illusory snakes. Collectively, we think that illusory snakes is due to a rivalry between the central and the peripheral retina; and their corresponding conscious brains; and the saccades are nothing but to convey parts of the retinal image from the center to the peripheries, and vice versa. Namely, the illusory snakes is generated by a spontaneous saccadic rivalry between the fovea $\&$ its corresponding conscious brain competing with the peripheral retina \& its corresponding conscious brain. Similarly, peripheral drift illusion that requires peripheral vision to be perceived, may not be generated without the aforementioned saccadic rivalry; namely, we think that the perception of that illusion may not be occurred without spontaneous saccade away from the fixational peripheral visual space, see also reference 1 and 5 . That saccade is mostly due to spatial attention which conveys the retinal image from the retinal peripheries (the fixational visual space) to the central retina (the attentional visual space). Namely, we think that without the aforementioned conveyance, the perceived illusion may not be generated because the aforementioned spatiotemporal discrepancies will be terminated. Importantly, we investigated the contribution of the human medial temporal complex in producing the illusory motion conscious perception with three different mechanisms: Cognitive control, deep breathing, and the arrangements of the patterns of the building blocks. The aforementioned processes are found to alter the visual perception of rotating snakes stimulus. Inclusively, we distinguished between two distinct visual awareness, namely, the central versus the peripheral vision and we show how active vision which requires cognitive control but not passive vision can ultimately control the perception of the rotating snakes stimulus, namely, alternation between real and illusory visual awareness.

Introduction \& Justification

Low contrast versions of an illusory rotating snakes image

(Kitaoka, 2009) inhibits the effect of illusory motion, see the supplementary materials (reference 3 ). To understand the aforementioned observation we have to be aware about the visual receptive field remapping which is a neurophysiological process to maintain visual stability during the saccadic eye movements; but since the stability may not be stand under certain conditions such as illusory snakes, we thus have to comprehend the following points:

1- Involuntary saccadic eye movements are reported to trigger illusory motion perception. For instance, microsaccade can cause displacement in the retinal image, indeed, its average peak velocity reaches to 28 degrees/sec (Otero-Millan, et al., 2012). The aforementioned displacement may convey parts of the retinal image from the fovea to its neighboring retinal peripheries. Importantly, these saccades must be spontaneous (involuntarily), namely, passive vision. If, however, that spontaneity is eliminated through cognitive control, namely, voluntary fixation or motion pursuit eye movements, the illusion could be ultimately suppressed. The suppression of the illusory snakes through cognitive control is assumed to be due to 'ultimately exclusive foveal receptive filed remapping', and rejections from the retinal peripheries to receive and/or process the external stimulus. In another word, active but not passive vision causes stoppage against the perception of illusory snakes because only the fovea is dealing with the external stimulus. We had run several fulsome experiments to validate the aforementioned statements. More importantly, the perception of the speed can be significantly reduced by decreasing the contrast, see reference 6 . This could be due to pupil constriction as a reflex to low contrast stimuli, which may disallow the retinal peripheries from signaling the brain. 2- The motion perception average thresholds are higher for deceleration in the fovea, and higher for acceleration in the retinal peripheries, Traschütz etal., 2012. Add to this, foveal 
cones takes about 30 milliseconds longer to signal the brain than the cones in the peripheral retina, Shina, 2017. Clearly, it appears that the visual perception agrees with the retinal physiology and its corresponding biological brains... 3- High contrast stimuli lead to pupil dilation, and low contrast stimuli lead to pupil constriction in both monkeys, and humans, Wang, et al., 2014. pupil dilation could be linked to rounded lens; therefore, more light rays may reach to the retinal peripheries, and pupil constriction could be linked to faltered lens, thus, most of the light rays will be focused in the fovea, (see reference 15). Pupil dilation may assist high contrast stimuli to easily access retinal peripheries, accordingly, the motion perception will speed up. However, the motion perception will slow down for low contrast stimuli maybe due to weaker access to retinal peripheries. Even with greater accessibility to retinal peripheries, if the stimulus has extremely low contrast; retinal peripheries may remain idle, see Robert Rosén, etal., 2014. Extremely low contrast therefore stops the perception of the illusory snakes because the conveyance between the perception of the fovea and the retinal peripheries is stopped, see supplementary martials. This is because the center-surround antagonism may not be able to motivate the retinal peripheries' ganglion cells to signal the brain, namely, those cells might be only generating very weak responses as products of imperfect lateral inhibition of the horizontal cells; signals that could not be noticed in the visual awareness. Because of these very weak retinal peripheries' signals; the brain might be requested to achieve two neural processes: The first neurological process might be; amplifying the aforementioned signals which may cause delays until these elements reach to the visual awareness (see reference 11 - to comprehend the meaning of visual awareness and where it might theoretically reside). The second neurological process possibly achieved, under extremely low contrast stimulus, the brain utilizes the visual memory in the parietal lobe (PL) to assist in perceptually filling-in those low contrast elements in the peripheries; processes that may cause additional temporal delays that allow the original signals from the fovea arrive to the visual awareness nearly simultaneously with the delayed signals from the peripheries, thus, the temporal discrepancies may be diminished, and thus, stoppage against the perception of illusory motion. Important to emphasize, this study mainly focus to show how the saccades, that convey parts of the retinal image from the central retina to the peripheral one and vice versa, namely saccadic rivalry, are the main reason of the perception of illusory snake, see supplementary martials. Namely, these transports trigger the two conscious brains' spatiotemporal discrepancies to work; see reference 2, and the supplementary materials. Before digging further in the neurophysiological analysis, in the following experiments, we shall investigate further parameters psychophysically to unionize the reported findings in regard to the perception of illusory snakes in the literature \& to optimize/utilize them, namely, touching the edges for better understanding of the fundamentals of the perception of illusory snakes! Materials \& Methods

Twelve human subjects, with dilated pupils, were recruited to participate in this study. Each stimulus subtends 30.96 DVA in length, and 18.77 DVA in width Each human subject has to undergo into four major trials; and each trail consisted of two different stimuli, a frame of reference (maximum strength of illusory motion), followed by a processed version of the frame of reference. We use a stimulus that produce extremely strong illusory snakes motion which had been created by Kitaoka in 2009; as an outstanding enhancement to his 2003 stimulus. We would like to emphasize that asymmetric repeated patterns of the four colored elements generate rotating snakes illusory motion; but symmetric patterns cannot generate RS illusory motion; for fulsome understanding, see the supplementary martials and reference 4. Each stimulus is presented for 10 seconds aiming for gathering the best subjective judgments that may eventually match with the physiological behaviors. The trials aim to collect statistical information from the twelve human subjects, namely, the average and the standard error of the mean as estimations of the illusory motion strength. It had been reported repeatedly that stable voluntary fixation significantly attenuates the illusory motion. In this study, however, our strict instructions were to 'ultimately' stare at the flickering star, see reference 3. As expected, when we instructed the human subjects to ultimate stare at the star, all of them had reported their ability to indefinitely null the perception of illusory motion, namely, they reported zero strength of illusory motion, as shown in the up-central infographic. Important to mention, a few human subjects had reported strain against the eye's muscles due to prolonged starring. Important to emphasize, despite the starring task is extremely easy to implement, however, it proves that the illusory motion perception is a product of spontaneous saccadic eye movements that shift a part of retinal image from the foveal perception to peripheral perception and vice versa, learn more about saccadic rivalry in reference 3; and supplementary materials. The illusion therefore emerges, if and only if, spatiotemporal discrepancies are emphasized due to the aforementioned shifting. It also had been reported that the overall contrast enhances the illusion, Backus etal. 2005. In another word, reduction against the overall contrast should inhibit the perception of illusory motion. To achieve this task, we had layered Kitaoka, 2009 stimulus with a dark gray layer that should convey the contrast between the white and black elements from value of 1 to 0.1 , it's $90 \%$ reduction, see 
reference 3. Same color contrast reduction is applied against the yellow and the blue elements. We requested from the twelve human subjects to compare the perception of illusory motion between the frame of reference against a globally extremely low contrast stimulus. All of the subjects had reported perception of zero illusory snakes perception for latter case; see the reasons in the previous section. The aforementioned experiment emphasizes that the perception of illusory motion can be inhibited when there is no spatiotemporal discrepancies between the central and the peripheral conscious brains, namely, when the peripheral conscious brain is almost off; no spatiotemporal discrepancies will exist, and thus stoppage against the illusory perception. For further validation to the spatiotemporal discrepancies hypothesis, we had to test the effect of blurriness. Backus etal. were the first to notice that blurring the rotating snakes images has effects on reducing the perception of illusory snakes. In this study, we firmly study their notice, but unlike starring, and low contrast trails, it's very noticeable that even with a massive blurriness, the illusion cannot be completely nulled, the reason will be discussed later in this section. Backus etal. had offered some explanations behind the effect of blurriness, namely, they think that in peripheral vision, blur decreases the illusion by removing high spatial frequencies from the neural image. However, in foveal vision, they think that blurring has an unmasking effect. We however had different perspective, because of its high level of uncertainty (uncertain decisive visual awareness), noisy/ blurred images seem to dilate the pupil size during the exposure, see reference 18. Uncertainty seems to require greater access to the 'recognitive memory' for better recognition, and we had been thinking about that retinal peripheries had been always responsible to activate the memory areas in the brain to generate fulsome visual awareness in uncertain circumstances. Pupil dilation, we think however, may accelerate the process of fulsome visual awareness; namely, when the pupil dilates, the anatomical lens tends to be more rounded to feed greater areas of retinal peripheries, see reference 15. In case of blurriness, the aforementioned retinal areas should be activated, in our opinion, to produce the best visual awareness of the external stimulus in a timely manner. Collectively, we cautiously do not fully agree with Bakus etal.'s explanation, namely, we think that the fovea might have a very minor function in the final creation of the visual awareness for the blurry/noisy RS images. In the last session of our experiments, we had asked the human subjects to pursuit a certain 'RS' circle in a massive 'RS' image, see the supplementary materials; aiming to discover the role of cognitive control on the perception of illusory motion. The twelve human subjects had reported a complete suppression against illusory motion in case of perfect smooth pursuit of the intended circle; however when the stimulus stopped with no smooth pursuit, all of them had reported to see it danced feverishly. The aforementioned reports confirm that the illusory motion can be triggered only under spontaneous saccadic eye movements; cognitive control, however, inhibit the illusion. To physiologize the previously collected behavioral data, we have to say, the central retina and the retinal peripheries do not have similar spatiotemporal properties, and their connections to the brain $\&$ to the visual awareness yields two distinct perceptions of the rotating snakes stimulus that trigger illusory motion perception. Namely, the illusory snakes emerges, if and only if, the 'two conscious brains' are active, namely, deactivating one of the previous brains partially or fully will suppress the strength of the perception of RS illusory motion partially or fully.

Asynchronized Respective Field Remapping.

Speculative Neural Network for Passive Vision:

Since the 'RS' illusion is a product of spontaneous saccadic eye movements, we therefore have to understand how the four ' $R S$ ' elements are processed in the brain in terms of receptive field remapping in the context of the two conscious brains. Important to emphasize, we hypothesized that each brain region has two distinct regions, including but not limited to the human medial temporal complex, see reference 19 , and 25. One of these two distinct regions is connected to the fovea, and another one connected to the retinal peripheries. Important to mention, the anatomical distinctions between each of these distinct regions in either the central or the peripheral conscious brains might be very hard achieved in some cases; namely, the neurons of each distinct region might be physical interweaved with other region! To deeply understand the illusory snakes perception, however, we have to understand the visual receptive field remapping, which is a great correction in vision research, Duhamel, 1992. It is about making the brain ready for taking new inputs from the future visual space, by utilizing a copy of corollary discharge "the signal that produced by superior colliculus and order the eye to execute the saccades" to the neocortex. Namely, a copy of corollary discharge has to be sent to the parietal lobe through PLVN (Ventral posterolateral nucleus), and to the frontal eye field area through MD (mediodorsal thalamus area), simultaneously with the original corollary discharge which has to be sent to the eye's muscles to execute a saccade. The hypothetically "peripheral parietal lobe", and its corresponding front eye field areas; are assumed to process the corollary discharge signal in different way than the neural processing manners for foveal receptive fields. The previous example shows how the 'two conscious brains' has different neural processing in almost every area in the brain.

Altogether, the holistic picture of visual field remapping might be theorized without violation to the concept of the 'two conscious brains' \& based on the temporal hierarchy that's gathered from the literature reviews, see figure 2 . The aforementioned circuit seems to be very sophisticated; but we think that it might be very close to the actual neural circuit for human visual motion awareness under involuntary saccadic eye movements despite that fact that most of the stimuli used in the literature have high contrast. Namely, in high contrast settings, the PL neuron should start to respond 80 milliseconds before the saccade, Duhamel, 1992, and this could be because of pupil dilation that led to rapid visual awareness due to 
greater access of retinal peripheries, see reference 15. Notice that the parietal lobe carries visual memory signals, and it is important to emphasize that intensive usage of that memory might delay the remapping process of the low contrast ' $R S$ ' elements (white and yellow elements). Namely, the PL will send visual information of the high contrast ' $R S$ ' elements to the following brain area (FEF) "an area that compresses the visual space before the saccade, Zirnsak, 2014" much faster; unlike the case of low contrast 'RS' elements. We therefore think that the partial lobe might have two distinct areas. Since the vivid visual awareness should be generated in synchronized harmony with the corresponding visual field remapping processes in the cortical network of vision; any delays in that process (caused by low contrast elements) might cause inaccurate spatial placement of these elements in the visual awareness, additionally; the size dilation will also cause additional spatial discrepancies, see reference 2 , and the supplementary materials (reference 3 ) for better understanding. To validate the aforementioned hypothesis, scientists may require reinstalling the Duhamel's experiment again, with visual stimuli that have different colors (blue, white, yellow, and black) on light gray background. White, and yellow visual stimuli are expected to show a delayed (PL) neural firing, with a greater amplitude. Different contrasts should produce different shapes of (PL) signals, in terms of onset, and peak amplitude. Namely, FEF will receive asynchronized (PL) signals form the different RS elements, and those signals might be merged within the 'compressed space' signals in first come first serve basis. The FEF 'merged' signal might be forwarded to $\mathrm{V} 4$, with spatiotemporal discrepancies due to the different four RS elements. Reportedly, high contrast stimuli promote the FEF neurons to be precisely activated at zero milliseconds and the activation peaks at 100 milliseconds after the beginning of the saccade. RS low contrast elements, which had not studied yet, are expected to trigger weaker signals from the FEF region; unless the peripheral FEF region is perfectly defined and localized. Because V4 remapping signal peaks at 200 milliseconds after the saccade, Neupane, etal. 2016b; we think that FEF may send its remapping signal to the hypothetical two distinct V4 areas that are assumed to process the sizes differently based on the contrast, in case of active peripheral V 4 area, to pursue the 'global' remapping process. The remapping signals afterwards might be sent to SC to decide whether there is motion, and forward the resultant signals to one of the two hypothetical two distinct hMT+ areas 'that should result in perceiving the velocities differently based on the contrast' before the creation of the illusory visual awareness; the illusory awareness happened just after the microsaccade, see OteroMillan, 2012. Needless to mention, microsaccades produce more frequent illusory snakes perception than saccades, namely, saccades but not microsaccades might have certain degrees to avoid some of asynchronized receptive filed remapping processes, and its resultant illusory perceptions.

Microsaccades but not the saccades seem to have greater capability to feed the retinal peripheries, thus, spatiotemporal discrepancies and illusory perception. We think that our interpretations may offer approaches to a generic neural network for human motion perception with free viewing conditions; however, we strongly urge the physiologists to reinstalling the Duhamel's experiment again, with visual stimuli that have different colors (blue, white, yellow, and black) on light gray background; and to converge the current available knowledge into one neural network, that may move very close to our imagined navigation as shown in figure 1. Unlike the previous brain imaging established experiments which exclusively examined the highest contrast stimuli and collected the signal from a single brain region; the future work should have sophisticated steps to examine the proposed neural network. In another word, all of the 'RS' four elements should be tested individually but collectively, using microelectrodes on animal models, preferably, macaque monkeys. The neurophysiological signals, however, must be collected simultaneously within certain time frame from all of the aforementioned brain regions using many microelectrodes to verify the network hierarchy. Ultimately, the concluding results may verify not only the secrets of the illusory motion perception, but it may explain how humans perceive motion under spontaneous saccadic eye movements, and the role of the two conscious brains in the creation of this illusion.

Essential Interpretations

We had discussed how to deactivate the retinal peripheries from signaling its corresponding conscious brain, through significant reduction against the stimuli contrast; however, deactivating the fovea, and its corresponding conscious brain seems to be very hard and illogical, because geometrically speaking, the probability that the light rays will not interact 
with fovea seems to be extremely low. Blurriness is known to dilate the pupil, and since the perception of illusory snakes had been significantly reduced through blurriness, see figure 1; this means there is very low discrepancies between the two conscious brains. We had been believing that the human pupil dilates its size for certain purposes, such as to allow more light rays to accessing the retinal peripheries, see reference 15; namely, we had been strongly disagreeing, based on previous studies that the pupil is just an opening to describe the cognitive functions; but these cognitive functions dilates the pupil for physical purposes. In another word, blurred images are preferred to be processed by the retinal peripheral, thus, pupil dilation that may activate the peripheral retina and its corresponding conscious brain; peripheral conscious brain, which we believe, it's an essential core of mental efforts \& processes. We therefore suggests two possible scenarios; either there is reasonable amount of the blurred stimulus' emitted photons could be exclusively entangled with retinal peripheries but not with the fovea; or the fovea but the retinal peripheries is completely inactivate (complete ignorance of the received light rays). Otherwise, the illusion will emerge due to the spatiotemporal discrepancies between the two integrative awareness generated by the fovea versus retinal peripheries. Comprehensive Navigations in Literature for Active Vision: The Engagement of Different Regions in the Prefrontal Cortex: Important to mention, we would like to inform the readers about the influence of deep breathing on the illusory motion, namely, we had discovered that deep inhalation diminishes the rotating snakes illusion, see reference 21 . Deep inhalation can be considered as a cognitive control process, and thus it could be settled under the umbrella of active vision. More interesting findings about active vision, we had recently confirmed that deep inhalation eliminates spatial wrapping, see reference 22; spatial wrapping that can be intensified by activating the motion detectors, see reference 23 . Consequently, cognitive control through deep inhalation "active vision" can inhibit the motion detectors from receiving illusory signals, thus, elimination against spatial wrapping. Neurophysiologically speaking, we theorized that the hMT+ region had two distinct areas, one is connected to the fovea and another is connected to the retinal peripheries, see reference 25 . We think that, at the end of deep inhalation; the oxygenated hemoglobin red blood cells might be significantly reduced in the brain; thus, just one out of the two aforementioned $\mathrm{hMT}+$ regions will have optimal neural cell respiration (optimal activation). Because the motion detectors are more common in the retinal peripheries, we think that deep inhalation deactivates the area that connected to the retinal peripheries but not the fovea. The aforementioned deactivation may disallow the emergence of spatiotemporal discrepancies; and therefore, the disappearance of the illusion. We therefore might disagree with Sirui Liu and his colleagues, see reference 24 , because we think that the human medial temporal complex is the main brain area that produces the perception of the illusory motion. Detailly speaking, we have shown that the repeated asymmetric patterns (RAP) stimuli are preferred to be processed in the retinal peripheries and its perceived speed is much greater than the repeated symmetric patterns stimuli; stimuli that might be preferred to be processed in the fovea, see reference 25 ; and this should explain why the controlled stimulus offered by Kitaoka had produced no illusory perception, see the supplementary materials. In another word, the perception of illusory snakes is disappeared from the controlled stimuli because it's built in a repeated symmetric patterns, and therefore, it may prefer to be processed by the fovea and there are mostly repulsive saccades preventing the light rays of that stimulus from feeding the retinal peripheries but the fovea. These repulsive saccades might be the main reason to terminate any spatiotemporal discrepancies between the fovea and the retinal peripheries, and thus complete stoppage against the perception of illusory snakes. We therefore believe that scientists should be very cautious when they compare 'controlled' stimuli which preferred to be processed by the fovea and its corresponding cortical regions with peripheral drift stimuli that preferred to jump from the fovea to the retinal peripheries, possibly through 'repulsive involuntary saccades' or 'pupil dilation' such as RAP stimuli or/and Gabor patch stimuli. Otherwise, the raw data of brain imaging might be greatly misrepresented. Important to mention, reduced BOLD signal doesn't mean idle brain area, but it might refer to fully functioning little area (central or peripheral) in the intended region. Conversely, high brain activities acquired by the conventional brain imaging techniques in a specific region shouldn't necessarily refer to direct responsibility, but it might refer to congested neural pathways; pathways that carry visual information to further brain areas that properly signal the visual awareness. Weirdly to suggest, high brain activities might 'sometimes' refer to undisciplined visual \& audiovisual awareness, possibly because when the central \& the peripheral 'conscious brains' are both active; rivalrous visual awareness may be produced unless voluntary hands' movements (active awareness) are applied, see reference 26 . Disciplined voluntary eye movements 'smooth pursuit' categorized as active visual awareness, and it seems to effectively stop the perception of illusory snakes, regardless the direction of the eye movements; namely, the aforementioned cognitive control process might allow one conscious brain (the foveal brain) but not the other to be active, process that show disallow the spatiotemporal discrepancies between the central \& peripheral conscious brain to occur. Important to mention, cognitive deep breathing was also an amazing controller of the rivalrous visual awareness; it has sufficient ability to switch between the central versus the peripheral vision, the real versus the illusion and the unwrapped versus the wrapped spaces, see reference, 21, 22, and 26 . The aforementioned facts show that we should clearly distinguish between active \& passive visual awareness, and to clearly states that the active controller of the visual awareness lies outside the visual cortex; possibly in the primary olfactory cortex but surely in the dorsolateral prefrontal cortex.

Markedly, the neural network we offered in this research is for passive vision, accordingly, we suspect the frontal eye field 
area instead of the dorsolateral prefrontal cortex to be a potential passive 'involuntary' controller of the spontaneous saccadic eye movements. In case of active vision, however, we suspect the dorsolateral prefrontal cortex to signal the frontal eye field with a desired signal to eventually create harmonical rivalrous vision. The role of dorsolateral prefrontal cortex, we believe, could be extended in every aspect in vision research; for instance, it had been reported that size-contrast illusions deceive the eye but not the hand, see reference 29; we however think that at the moment of actuation (grasping) in active vision situations, the eye $\&$ the hand of human subjects might not be deceived.

The Oval Rotating Snakes Planet

Finally, we would like to share with the readers a little artistic work that also carries scientific meanings, we call it "the Oval Rotating Snakes Planet". We created that planet to informally explore the role of attentional shifts towards the peripheral visual spaces in eliminating the Rotating Snakes illusion. Truncating these silent objects in the peripheries visual spaces may trigger RS illusory motion, mainly because the fovea will not be occupied by those silent objects and thus spatiotemporal discrepancies (rivalry between the fovea and the retinal peripheries for RS stimulus). Keeping these objects may suppress the RS illusory motion; because the fovea will be occupied, and thus minimal spatiotemporal discrepancies.

Essential Notifications

Important to emphasize, readers should have direct access to the stimuli, see reference 3 . Advisably, download the videos for the best quality. Watch the videos in moderate brightness, if the observer is an elder adult however, noticing the illusion will be somehow hard because human's pupil normally constricts with age and thus the stimulus may stay exclusively within the macula region, see reference 27 and 28. In another word, there will be weak spatiotemporal discrepancies between the foveal and the peripheral retinal 'conscious' images. Since caffeine intake dilate the pupil, in case of pinpoint pupils, see reference 20 , we strongly advise to drink a cup of coffee before the experiments. In the end of our letter, we would like to inform scholars with brain imaging facilitations that we welcome to collaboration in challenging investigations, namely, to achieve critical analyses of the spatiotemporal properties of the aforementioned two distinct conscious brains. Enquiries may be sent to the author.
Transactional References

[1] Fraser, \& Wilcox (1979). "Perception of illusory movement". Nature. [2] Yousef, 2019. "Spatial Attention Dilates Time and Length." PsyArXiv.

[3] You may find the stimuli in the following link: https://drive.google.com/drive/folders/1YrAUT_WrzbK73_KIK_WLNiZa-8mpE_7w [4] Kuriki, I. Ashida, H. Murakami, I. Kitaoka, A. (2008). Functional brain imaging of the 'Rotating Snakes' illusion. Journal of Vision.

[5] Otero-Millan, etal. (2012). Microsaccades and Blinks Trigger Illusory Rotation in the Rotating Snakes Illusion. J Neuroscience.

[6] Thompson (1982). Perceived rate of movement depends on contrast. Vision Research. [7] Traschütz etal., 2012. Speed change detection in foveal and peripheral vision. Vision Research.

[8] Sinha, etal. (2017). Cellular and circuit mechanisms shaping the perceptual properties of the primate fovea. Cell 168, 413-426.

[9] Wang, et al., (2014). Modulation of stimulus contrast on the human pupil orienting response. EJN, 2014.

[10] R. Rosén; etal. (2014) Quick contrast sensitivity measurements in the periphery, in Journal of Vision,

[11] Yousef, Ahmad. 2019. "Consciousness Might Be Localized in Extra Physical Dimensions." PsyArXiv. 10.31234/osf.io/angc8.

[12] Duhamel, 1992. The updating of the representation of visual space in parietal cortex by intended eye movements. Science Mag.

[13] Zirnsak, etal., 2014. Visual space is compressed in prefrontal cortex before eye movements. Nature.

[14] Neupane et al. 2016. Dissociation of forward and convergent remapping in primate visual cortex. Current Biology.

[15] Yousef, Ahmad. 2019. "Linking Dynamics Between Pupil and Lens." engrXiv. doi:10.31224/osf.io/9c3vk

[16] Tomimatsu, etal., (2011). Halt and recovery of illusory motion perception from peripherally viewed static images. Attention, Perception, \& Psychophysics.

[17] B. Backus; i. Oruç (2005). Illusory motion from change over time in the response to contrast and luminance. Journal of Vision.

[18] Urai, etal., (2017). Pupil-linked arousal is driven by decision uncertainty and alters serial choice bias. Nature Communications

[19] Yousef, Ahmad. 2019. "Retinal Peripheries Generate Illusory Motion Reversals." PsyArXiv. doi:10.31234/osf.io/gfmpe.

[20] Abokyi, etal.,(2017). Caffeine intake is associated with pupil dilation and enhanced accommodation. Eye (Lond) Journal.

[21] Yousef, Ahmad. 2019. “Deep Breathing Alters Visual Motion Perception.” PsyArXiv. doi:10.31234/osf.io/up3sa.

[22] Yousef, Ahmad. 2019. "Deep Inhalation Eliminates Spatial Wrapping." PsyArXiv. doi:10.31234/osf.io/wgjy7.

[23] Vaughn and Eagleman. (2013). Spatial warping by oriented line detectors can counteract neural delays. Front Psychology

[24] Liu, etal (2019) Neural Correlates of the Conscious Perception of Visual Location Lie Outside Visual Cortex. Current Biology.

[25] Yousef, A. 2020. "Two Distinct Human Medial Temporal Complexes." PsyArXiv. doi:10.31234/osf.io/8dzk

[26] Yousef, A. 2020. "Voluntary Movements Discipline Audiovisual Perception." PsyArXiv. doi:10.31234/osf.io/bqr6u.

[27] Sloane, etal. 1988. Aging, senile miosis and spatial contrast sensitivity at low luminance. Vision Research.

[28] Billino, 2009. Age Effects on Perception of Motion Illusions. Perception. [29] Aglioti, 1995. Size-contrast illusions deceive the eye but not the hand. Current Biology. 